\title{
$\mathrm{BMJ}$
}

\section{Antenatal education and postnatal support strategies for improving rates of exclusive breast feeding: randomised controlled trial}

\author{
Lin-Lin Su, associate consultant, ${ }^{1}$ Yap-Seng Chong, senior consultant, ${ }^{1}$ Yiong-Huak Chan, head, biostatistics \\ unit, ${ }^{2}$ Yah-Shih Chan, assistant director of nursing, ${ }^{3}$ Doris Fok, research coordinator and lactation \\ consultant, ${ }^{3}$ Kay-Thwe Tun, clinical project coordinator, ${ }^{4}$ Faith S P Ng, biostatistician, ${ }^{4}$ Mary Rauff, senior \\ consultant $^{1}$
}

Department of Obstetrics and Gynaecology, Yong Loo Lin School of Medicine, National University of Singapore, Singapore 119074

${ }^{2}$ Yong Loo Lin School of Medicine, National University of Singapore, Singapore 117597

${ }^{3}$ Department of Obstetrics and Gynaecology, National University Hospital, Singapore 119074

${ }^{4}$ Clinical Trials and Epidemiology Research Unit (CTERU), Singapore 1699039

Correspondence to: Y-S Chong obgcys@nus.edu.sg

doi:10.1136/bmj.39279.656343.55

\section{ABSTRACT}

Objective To investigate whether antenatal breast feeding education alone or postnatal lactation support alone improves rates of exclusive breast feeding compared with routine hospital care.

Design Randomised controlled trial.

Setting A tertiary hospital in Singapore.

Participants 450 women with uncomplicated pregnancies.

Main outcome measures Primary outcomes were rates of exclusive breast feeding at discharge from hospital and two weeks, six weeks, three months, and six months after delivery. Secondary outcomes were rates of any breast feeding.

Results Compared with women who received routine care, women in the postnatal support group were more likely to breastfeed exclusively at two weeks (relative risk 1.82, $95 \%$ confidence interval 1.14 to 2.90 ), six weeks (1.85, 1.11 to 3.09$)$, three months $(1.87,1.03$ to 3.41$)$, and six months $(2.12,1.03$ to 4.37$)$ postnatally. Women receiving antenatal education were more likely to breast feed exclusively at six weeks $(1.73,1.04$ to 2.90$)$, three months $(1.92,1.07$ to 3.48$)$, and six months $(2.16,1.05$ to 4.43$)$ postnatally. The numbers needed to treat to achieve one woman exclusively breast feeding at six months were 11 ( 6 to 80 ) for postnatal support and 10 (6 to 60) for antenatal education. Women who received postnatal support were more likely to exclusively or predominantly breast feed two weeks after delivery compared with women who received antenatal education $(1.53,1.01$ to 2.31$)$. The rate of any breastfeeding six weeks after delivery was also higher in the postnatal support group compared with women who received routine care $(1.16,1.02$ to 1.31$)$.

Conclusions Antenatal breast feeding education and postnatal lactation support, as single interventions based in hospital both significantly improve rates of exclusive breast feeding up to six months after delivery. Postnatal support was marginally more effective than antenatal education.

Trial registration Clinical Trials NCT00270920.

\section{INTRODUCTION}

Despite awareness of the many advantages of breast feed ing, its rates often fall short of recommended practice. The World Health Organization ${ }^{1}$ and the American Academy of Pediatrics ${ }^{2}$ advocate exclusive breast feeding for six months and partial breast feeding thereafter for at least 12 or 24 months. In an effort towards achieving better breast feeding practices, UNICEF and WHO launched the baby friendly hospital initiative in 1991 to ensure that all maternity facilities support mothers in making the best choice about feeding. The initiative was introduced to the United Kingdom in 1993, but, although improvements have been reported ${ }^{3}$ rates of breast feeding in the UK are still among the lowest in the world. ${ }^{45}$ Recent reports from the National Institute for Health and Clinical Excellence (NICE) urge NHS units to become baby friendly to improve rates of breast feeding and save money. ${ }^{46}$ Data from the millennium cohort study, however, show that though participating maternity units in the UK increased rates of initiation of breastfeeding, duration did not increase. ${ }^{5}$ Other strategies are therefore required to support mothers in the UK to breast feed for the recommended time. The challenge lies in implementing programmes that can effectively improve rates of short and long term exclusive breast feeding.

A national survey in Singapore in 2001 found that only $21 \%$ of mothers were breast feeding at six months, with less than $5 \%$ of mothers exclusively breast feeding, despite the fact that nearly $90 \%$ of the mothers surveyed indicated that breast feeding was the best form of infant nutrition and 95\% said they had attempted to breastfeed. ${ }^{7}$ It is evident that many mothers are unable to establish and maintain breast feeding successfully, despite wanting to do so. While antenatal education and counselling is helpful, ${ }^{8} 68 \%$ of mothers said that early problems with breast feeding was the main reason they stopped nursing before two months postpartum. ${ }^{7}$ Other barriers were lack of knowledge about breast feeding and lack of support from health professionals. $^{7}$ Women value being shown how to breast feed rather than being told how to. ${ }^{910}$ Evidence of effective interventions to improve exclusive breast 
feeding for the recommended duration of six months is sparse. While there is evidence for the effectiveness of professional support in prolonging duration of breast feeding and increasing rates of initiation of breast feeding, the strength of its effect on the rate of exclusive breastfeeding is unclear. ${ }^{11} 12$

We used a randomised controlled study to compare the relative effectiveness of an antenatal breast feeding education protocol and a postnatal lactation support protocol versus routine care in improving rates of exclusive breast feeding in a tertiary hospital setting.

\section{METHODS}

\section{Study population}

We recruited healthy pregnant women who were attending antenatal clinics at the National University Hospital, a tertiary hospital in Singapore. One research assistant, who is an experienced lactation consultant, recruited women from the outpatient obstetric clinic. Mothers were eligible for participation if they were more than 34 weeks' gestation at the time of delivery, expressed an intention to breast feed, and had no illness that would contraindicate breast feeding or severely compromise its success. We excluded women with high risk and multiple pregnancies. Women who agreed to participate gave written informed consent.

\section{Definitions of types of breastfeeding}

Exclusive breast feeding-only breast milk given to baby. Medicines, vitamins, and oral rehydration solution may be given but no formula or water Predominant breast feeding-breast milk and water, sweetened water, and juices given without formula

Partial breast feeding-breast milk and complementary food such as formula milk, gruel, semisolids, or solids are given

No breast feeding - no breast milk given and only formula milk and other liquids or food given

\section{Assignment and intervention}

Women were randomised into three groups. Group 1 was the control group and women received routine antenatal, intrapartum, and postnatal obstetric care with no special intervention applied. At our hospital, this included optional antenatal classes, which did address infant feeding, and postnatal visits by a lactation consultant should any problems with breast feeding arise.

Women randomised to group 2 received one session of antenatal breastfeeding education in which they were shown a 16 minute educational video entitled "14 Steps to Better Breastfeeding” (InJoy Videos, Boulder, CO), which introduced the benefits of breastfeeding, demonstrated correct positioning, latch on, and breast care, and

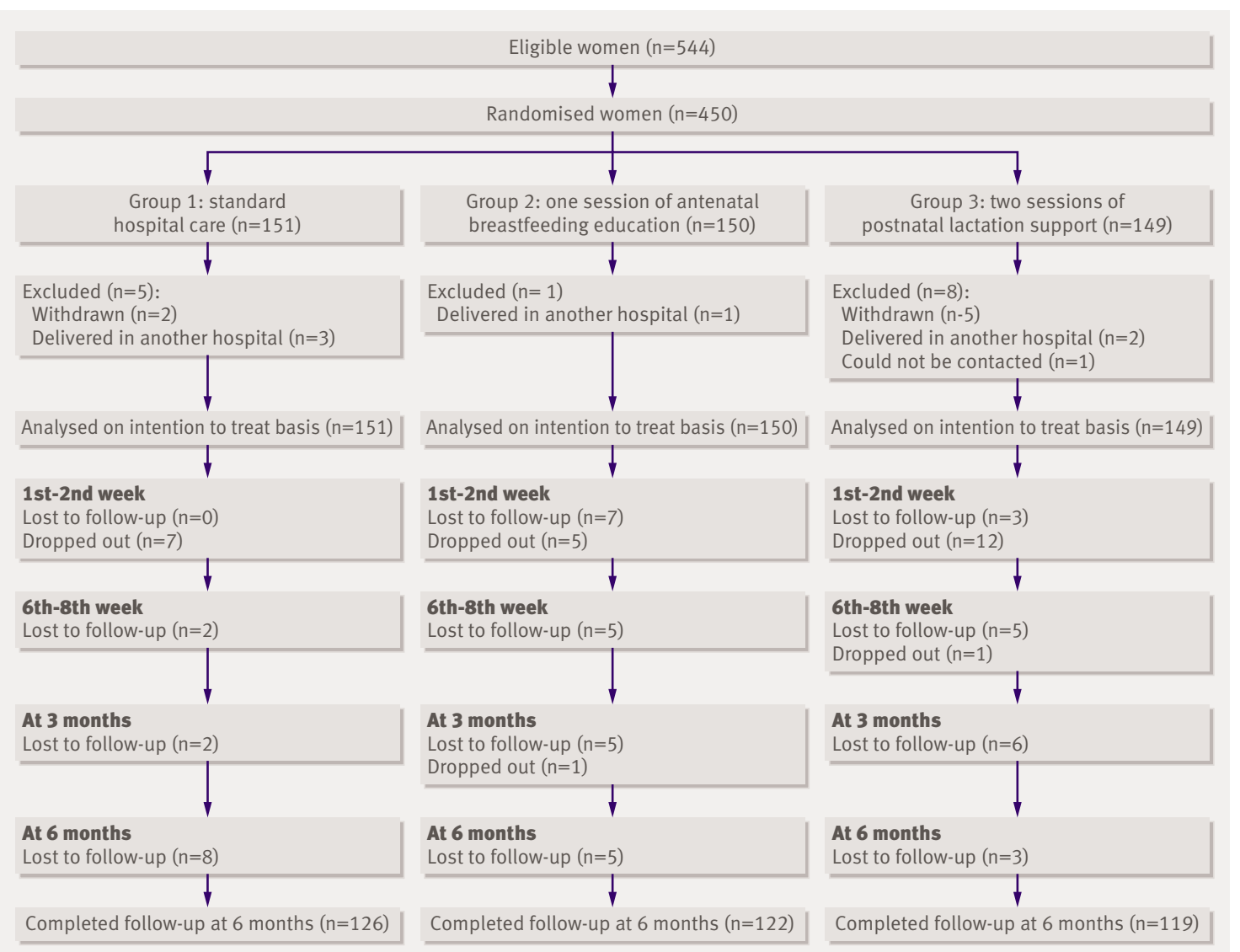


discussed common concerns. They were also given printed guides on breast feeding ${ }^{1314}$ and an opportunity to talk to a lactation counsellor for about 15 minutes. They subsequently received routine intrapartum and postnatal obstetric care.

Women randomised to group 3 were placed in a two session postnatal lactation support programme. They were visited by a lactation consultant within the first three postnatal days before discharge from hospital. They also received the same printed guides on breast feeding ${ }^{1314}$ during this visit. A second support session was provided during their first routine postnatal visit one to two weeks after delivery. During these two encounters, the women received hands-on instructions in latching on, proper positioning, and other techniques to avoid common complications. Each encounter lasted about 30 minutes.

We conducted our study in conjunction with the clinical trials and epidemiology research unit, which is an independent organisation funded by the National Medical Research Council. This unit performed the randomisation, sequence allocation, trial coordination, site monitoring, data collection, and analysis for this study according to good clinical practice guidelines. The unit generated and maintained a list of random codes for participants, corresponding to the two interventions and the control assignment groups. Treatment assignment was generated with a computer programme. The clinical project coordination department of the Clinical Trials and Epidemiology Research Unit randomised women by means of telephone calls. Unit personnel would then log on to the password protected website to obtain the randomisation number and assign the study group. Backup envelopes were used if website randomisation failed. The sequence was therefore strictly concealed until the intervention was assigned. The research assistant ensured that appropriate interventions were carried out depending on the group to which the women were allocated. The trial data were collected on printed case record forms, and the unit performed data entry. Clinical project coordinators of the unit regularly monitored sites to ensure accuracy of recruitment and data collection as well as strict compliance to the study protocol. We

\begin{tabular}{|c|c|c|c|c|}
\hline & Group $1(\mathrm{n}=151)$ & Group $2(n=150)$ & Group $3(n=149)$ & Total $(n=450)$ \\
\hline Mean (SD) age (years) & $28.6(5.8)$ & $29.5(5.2)$ & $29.9(6)$ & $29.4(5.6)$ \\
\hline \multicolumn{5}{|l|}{ Parity: } \\
\hline Primiparous & $60(40)$ & $59(39)$ & $59(40)$ & $178(40)$ \\
\hline Multiparous & $91(60)$ & $91(61)$ & $90(60)$ & $272(60)$ \\
\hline \multicolumn{5}{|l|}{ Ethnicity: } \\
\hline Chinese & $46(31)$ & $62(41)$ & $65(44)$ & $173(38)$ \\
\hline Malay & $82(54)$ & $65(43)$ & $69(46)$ & $216(48)$ \\
\hline Indian & $16(11)$ & $20(13)$ & $12(8)$ & $48(11)$ \\
\hline Other & $7(5)$ & $3(2)$ & $3(2)$ & $13(3)$ \\
\hline \multicolumn{5}{|c|}{ Highest educational qualification: } \\
\hline Higher than secondary & $53(35)$ & $56(37)$ & $51(34)$ & $160(36)$ \\
\hline No qualification/primary & $98(65)$ & $94(63)$ & $98(66)$ & $290(64)$ \\
\hline \multicolumn{5}{|l|}{ Employment: } \\
\hline Student/housewife & $86(57)$ & $86(57)$ & $69(46)$ & $241(54)$ \\
\hline Employed & $65(43)$ & $64(43)$ & $80(54)$ & $209(46)$ \\
\hline \multicolumn{5}{|c|}{ Entitlement to $>2$ months maternity leave for employed women: } \\
\hline Yes & $27(42)$ & $26(41)$ & $31(39)$ & $84(40)$ \\
\hline No & $31(48)$ & $31(48)$ & $40(50)$ & $102(49)$ \\
\hline Not applicable & $7(11)$ & $7(11)$ & $9(11)$ & $23(11)$ \\
\hline \multicolumn{5}{|l|}{ Household monthly income: } \\
\hline «Singapore $\$ 5000$ & $141(93)$ & $132(88)$ & $136(91)$ & $409(91)$ \\
\hline$\geq$ Singapore $\$ 5000$ & $10(7)$ & $18(12)$ & $13(9)$ & $41(9)$ \\
\hline \multicolumn{5}{|l|}{ Family structure: } \\
\hline Nuclear & $80(53)$ & $81(54)$ & $68(46)$ & $229(51)$ \\
\hline Not nuclear & $71(47)$ & $69(46)$ & $81(54)$ & $221(49)$ \\
\hline \multicolumn{5}{|l|}{ Had previously breast fed: } \\
\hline Yes & $85(56)$ & $85(57)$ & $84(56)$ & $254(56)$ \\
\hline No & $66(44)$ & $65(43)$ & $65(44)$ & $196(44)$ \\
\hline \multicolumn{5}{|c|}{ Attended hospital antenatal class: } \\
\hline Yes & $7(5)$ & $12(8)$ & $9(6)$ & $28(6)$ \\
\hline No & $144(95)$ & $138(92)$ & $140(94)$ & $422(94)$ \\
\hline
\end{tabular}


recorded and discussed all instances of protocol violation. Similarly, a research assistant recorded, and the unit monitored, all adverse events. All data were kept confidential and analysis was not performed until completion of the study.

\section{Outcome measures}

The primary outcomes were rates of exclusive breast feeding at discharge from the hospital and at two weeks, six weeks, three months, and six months after delivery. We defined exclusive breast feeding as giving breast milk as the only food source, with no other foods or liquids, other than vitamins or medications, being given. The box shows the definitions of the four categories of breast feeding. Secondary outcomes were the frequencies of any breast feeding at each of these intervals.

\section{Follow-up}

During the baseline antenatal interview, all mothers answered a standard questionnaire that documented their demographic data, home environment, and experience of breast feeding. They were also given an infant feeding diary. The first postnatal interview was conducted before the women were discharged from the hospital. Detailed data about the intrapartum and immediate postpartum experience, including mode of delivery, birth weight of newborns, and infant feeding in the hospital, were recorded during this interview. The two week and six week interviews were performed either during the women's routine clinic visit for postnatal reviews or via home visits. At these visits, they were asked to fill in a standard questionnaire regarding infant feeding by referring to their diaries. The mothers were subsequently interviewed over the telephone at three months and six months after delivery regarding their breast feeding and weaning practices as recorded in their infant feeding diaries. Rates of exclusive, predominant, partial, and no breast feeding were tracked at all these time points.

\section{Statistical analysis}

To calculate sample size, we estimated that at six months $10 \%$ in group $1,15 \%$ in group 2 , and $25 \%$ in group 3 would still be breast feeding. To detect these differences across the three groups with a two sided test

\begin{tabular}{|c|c|c|c|c|}
\hline & $\begin{array}{l}\text { Group } 1 \\
(n=138)\end{array}$ & $\begin{array}{l}\text { Group } 2 \\
(n=138)\end{array}$ & $\begin{array}{l}\text { Group } 3 \\
(n=134)\end{array}$ & $\begin{array}{c}\text { Total } \\
(n=410)\end{array}$ \\
\hline \multicolumn{5}{|l|}{ Mode of delivery: } \\
\hline Normal vaginal & $105(76)$ & $104(75)$ & $103(77)$ & $312(76)$ \\
\hline Vacuum (ventouse) & $3(2)$ & $3(2)$ & $4(3)$ & $10(3)$ \\
\hline Forceps & 0 & 0 & $1(1)$ & $1(0.2)$ \\
\hline Caesarean section & $30(22)$ & $31(22)$ & $26(19)$ & $87(21)$ \\
\hline Mean (SD) gestational age at birth (weeks) & $39.1(1.3)$ & $39.2(1.2)$ & $39.4(1.3)$ & $39.2(1.3)$ \\
\hline Mean (SD) birth weight (g) & 3194 (439) & $3171(429)$ & $3171(411)$ & $3179(426)$ \\
\hline
\end{tabular}

*Group 1=standard hospital care; group 2=antenatal breastfeeding education; group 3=postnatal lactation support. Based on number of women who delivered at the hospital. of $5 \%$ with $90 \%$ power we needed to randomise 450 women equally into the three groups.

The trial data were entered into CLINTRIAL version 4.4 (PhaseForward), specialised software for managing longitudinal trial data. This programme facilitates interactive entry and data correction and maintains consistent and accurate trial data. We used SAS version 9.1 (SAS Institute, Cary, NC, USA) for statistical analyses. We collected descriptive statistics on the breast feeding for the three groups and analysed data on an intention to treat basis. We assessed the pairwise comparisons between the different study groups in their rates of breastfeeding using modified Cox regression analysis ${ }^{15}$ to provide the adjusted relative risks and 95\% confidence intervals. Significance was set at $\mathrm{P}<0.05$. We carried out primary analyses for all participants who had completed follow-ups, with sensitivity analyses when appropriate.

\section{RESULTS}

We recruited 450 women from February 2004 to September 2005, of whom 151 were randomised to receive standard hospital care (group 1), 150 to antenatal education (group 2), and 149 to postnatal lactation support (group 3). Four women were randomised by using backup envelopes because of dysfunction in web randomisation and this resulted in the imbalance in numbers of women per group. Follow-up was completed in May 2006. The figure shows the trial profile, including the number of women lost to follow-up. In total, 367 $(82 \%)$ completed six months of follow-up, with a similar number lost to follow-up in the three study groups. Baseline characteristics among the three randomised groups were similar (table 1). The three study groups were also similar in the variables related to birth and infant morbidity, including the mode of delivery and the mean birth weight (table 2).

\section{Effect of intervention}

Table 3 shows the primary outcome of rates of exclusive breast feeding at the various time points for each group. Compared with the control group, women randomised to postnatal intervention were significantly more likely to breast feed exclusively from two weeks till six months after delivery. At two weeks, 38\% (48/ 128) of women randomised to postnatal intervention were exclusively breast feeding compared with $21 \%$ (28/136) of women who received routine hospital care (relative risk 1.82; 95\% confidence interval 1.14 to 2.90 ; number needed to treat $=6,4$ to 17 ). This significant improvement was still present six weeks, three months, and six months after delivery (table 3). At six months, 19\% (22/119) of women in the postnatal intervention group were exclusively breastfeeding compared with $9 \%(11 / 126)$ of the women in the control group $(2.12 ; 1.03$ to 4.37$)$. For every 11 women who received postnatal lactation support, one exclusively breast fed for six months (number needed to treat $=11,6$ to 80 ).

Women randomised to antenatal education were more likely to exclusively breast feed compared with 
the control group only from six weeks postnatally, when $29 \%(39 / 133)$ of women in the antenatal education group were exclusively breastfeeding compared with $17 \%(23 / 136)$ of women receiving routine care $(1.73,1.04$ to 2.90 ; number needed to treat $=8,5$ to 41). This significant benefit was also evident at three months and six months after delivery (table 3). At six months, $19 \%$ (23/122) of women randomised to receiving antenatal education were exclusively breastfeeding compared with $9 \%(11 / 126)$ of women in the control group $(2.16 ; 1.05$ to 4.43$)$. One woman exclusively breast fed for six months for every 10 women who received antenatal breastfeeding education (number needed to treat $=10,6$ to 60 ).

We compared the efficacy of antenatal education and postnatal support with regard to breast feeding and found no significant difference in improvements in the rate of exclusive breast feeding (table 3). However, women who received postnatal support were more likely to either exclusively or predominantly breastfeed their babies at two weeks compared with women who received antenatal education $(1.53,1.01$ to 2.31 ; number needed to treat $=7,4$ to 28 ).

We also assessed the secondary outcome of the rate of any breast feeding. The incidence of any breast feeding was higher in women who received postnatal lactation support than in women in the control group (1.19, 1.05 to 1.36 ; number needed to treat $=8,5$ to 26 ) at six weeks after delivery (table 4 ). They were also more likely to breast feed at six weeks compared with women who received antenatal education $(1.16,1.02$ to 1.31 ; number needed to treat $=9,5$ to 60 ). There was no significant difference among the three groups at discharge from hospital, two weeks, three months, and six months after delivery.

\section{Sensitivity analysis}

Our primary data analysis was based on women who completed follow-up at the particular time points of data collection. The main reason for loss to follow-up was that we could not contact the women. We performed sensitivity analyses on the assumption that none of the women lost to follow-up were exclusively breast feeding at any time point. With these assumptions, women who received antenatal education were significantly more likely to be exclusively breast feeding at six weeks $(1.71,1.02$ to 2.86$)$, three months (1.84, 1.02 to 3.32$)$, and six months $(2.11,1.03$ to 4.32$)$ compared with the women receiving routine care. Women who received postnatal lactation support were also more likely to exclusively breast feed at two weeks $(1.74,1.09$ to 2.77$)$ and six weeks $(1.76,1.06$ to 2.94$)$ compared with the control group.

\section{DISCUSSION}

Antenatal breastfeeding education and postnatal lactation support both significantly improved the rates of exclusive breastfeeding up to six months after delivery compared with routine care in a tertiary hospital setting. While both strategies were effective, postnatal support was marginally more effective than antenatal education in improving breastfeeding practice.

\section{Strengths and weaknesses}

This study was rigorously conducted. All the mothers in our study complied with the intervention. Compliance with the assigned interventions was documented in the case record files and monitored by clinical project coordinators. We minimised potential recall bias in maternal self reporting of breastfeeding with infant feeding diaries. Though the study was pragmatic and carried out in a non-research setting in a busy tertiary hospital, we were able to follow good clinical practice guidelines. Women received both antenatal and postnatal interventions in addition to routine ambulatory and inpatient hospital care. All other aspects of management were similar. The findings can therefore be generalised to any setting where women's pregnancy and delivery are managed in a hospital setting. Our primary outcome was rates of exclusive breast feeding up to six months after delivery. The protective effects of breast feeding have been shown to be dose responsive ${ }^{16-18}$ and minimal breast feeding may not be protective. ${ }^{17}$ Researchers in lactation have advocated that research on promotion of breast feeding must target exclusive breast feeding, ${ }^{19}$ and ours is one of the larger randomised controlled trials with this primary outcome.

Most of the women in our study did not attend the optional antenatal classes offered by the hospital. Our

\begin{tabular}{|c|c|c|c|c|c|c|}
\hline & \multirow[b]{2}{*}{ Group 1} & \multirow[b]{2}{*}{ Group 2} & \multirow[b]{2}{*}{ Group 3} & \multicolumn{3}{|c|}{ Relative risk $(95 \% \mathrm{Cl})$; number needed to treat $(\mathrm{NNT})(95 \% \mathrm{Cl})$} \\
\hline & & & & Group $2 v$ group 1 & Group $3 v$ group 1 & Group $3 v$ group 2 \\
\hline $\begin{array}{l}\text { At discharge from } \\
\text { hospital }\end{array}$ & $25 / 138(18)$ & $27 / 138(20)$ & $36 / 134(27)$ & 1.08 (0.63 to 1.86 ), $\mathrm{P}=0.782$ & 1.48 ( 0.89 to 2.47$), P=0.130$ & 1.37 (0.83 to 2.26$), P=0.213$ \\
\hline At 2 weeks & $28 / 136(21)$ & $36 / 133(27)$ & $48 / 128(38)$ & 1.32 (0.80 to 2.15$), P=0.278$ & $\begin{array}{c}1.82(1.14 \text { to } 2.90), P=0.012 ; \\
\text { NNT=6 ( } 4 \text { to } 17)\end{array}$ & 1.39 (0.90 to 2.13$), P=0.139$ \\
\hline At 6 weeks & 23/136 (17) & $39 / 133(29)$ & 40/128(31) & $\begin{array}{c}1.73(1.04 \text { to } 2.90), P=0.036 ; \\
\text { NNT=8 (5 to } 41)\end{array}$ & $\begin{array}{c}1.85 \text { (1.11 to } 3.09), P=0.019 ; \\
\text { NNT=7 ( } 4 \text { to } 24)\end{array}$ & 1.07 (0.69 to 1.66 ), $P=0.777$ \\
\hline At 3 months & $17 / 134(13)$ & $31 / 127(24)$ & $29 / 122(24)$ & $\begin{array}{c}1.92(1.07 \text { to } 3.48), P=0.030 ; \\
\text { NNT=9 ( } 5 \text { to } 43)\end{array}$ & $\begin{array}{c}1.87 \begin{array}{c}(1.03 \text { to } 3.41), P=0.040 \\
\text { NNT }\end{array}=9(5 \text { to } 60)\end{array}$ & 0.97 (0.59 to 1.62$), P=0.918$ \\
\hline At 6 months & $11 / 126(9)$ & 23/122 (19) & $22 / 119$ (19) & $\begin{array}{c}2.16(1.05 \text { to } 4.43), P=0.036 ; \\
\text { NNT }=10(6 \text { to } 60)\end{array}$ & $\begin{array}{c}2.12(1.03 \text { to } 4.37), P=0.042 ; \\
N N T=11(6 \text { to } 80)\end{array}$ & 0.98 (0.55 to 1.76$), P=0.948$ \\
\hline
\end{tabular}

*Group 1=standard hospital care; group 2=antenatal breastfeeding education; group 3=postnatal lactation support. Based on completed follow-up. 
Table 4 | Number (percentage) of women breastfeeding at all by group allocation*

\begin{tabular}{|c|c|c|c|c|c|c|}
\hline & \multirow[b]{2}{*}{ Group 1} & \multirow[b]{2}{*}{ Group 2} & \multirow[b]{2}{*}{ Group 3} & \multicolumn{3}{|c|}{ Relative risk $(95 \% \mathrm{Cl})$; number needed to treat (NNT) $(95 \% \mathrm{CI})$} \\
\hline & & & & Group $2 v$ group 1 & Group $3 v$ group 1 & Group $3 v$ Group 2 \\
\hline At discharge from hospital & $131 / 138(95)$ & $132 / 138(96)$ & $131 / 134(98)$ & 1.01 (0.79 to 1.28$), P=0.951$ & 1.03 (0.81 to 1.31$), P=0.812$ & $1.02(0.80$ to 1.30$), P=0.860$ \\
\hline At 2 weeks & $127 / 136(93)$ & $126 / 133(95)$ & $126 / 128(98)$ & 1.02 (0.79 to 1.20$), P=0.909$ & 1.05 (0.82 to 1.35$), P=0.675$ & 1.04 (0.81 to 1.33$), P=0.761$ \\
\hline At 6 weeks & $96 / 136(71)$ & $97 / 133(73)$ & $108 / 128(84)$ & 1.03 (0.89 to 1.20$), P=0.669$ & $\begin{array}{c}1.19(1.05 \text { to } 1.36), P=0.008 ; \\
\text { NNT=8 ( } 5 \text { to } 26)\end{array}$ & $\begin{array}{c}1.16(1.02 \text { to } 1.31), P=0.024 ; \\
N N T=9(5 \text { to } 60)\end{array}$ \\
\hline At 3 months & $65 / 134(49)$ & $73 / 127(58)$ & $71 / 122(58)$ & 1.19 (0.85 to 1.66$), P=0.320$ & 1.20 (0.86 to 1.68$), P=0.289$ & 1.01 (0.73 to 1.40$), P=0.941$ \\
\hline At 6 months & $43 / 126(34)$ & $52 / 122(43)$ & $48 / 119(40)$ & 1.25 (0.83 to 1.87$), P=0.281$ & 1.18 (0.78 to 1.78$), P=0.426$ & 0.95 (0.64 to 1.40$), P=0.783$ \\
\hline
\end{tabular}

*Group 1=standard hospital care; group 2=antenatal breastfeeding education; group 3=postnatal lactation support. Based on completed follow-up.

results may not apply to settings where advice on breast feeding or attendance at antenatal classes is part of standard hospital care. The rates of any and exclusive breastfeeding in our control population (group 1) were relatively low at only $34 \%$ and $9 \%$, respectively, six months after delivery. Our findings may not be applicable in settings where the baseline breastfeeding practice is better. Statistics from the Infant Feeding 2000 survey, however, suggest that rates of breast feeding in the $\mathrm{UK}^{20}$ are similar to those of Singapore. ${ }^{7}$ Our results were also consistent with the results of PROBIT study in Belarus, in which $36 \%$ of women in the control group were breast feeding at all at six months. ${ }^{21}$ Around $90 \%$ of the women in our study had monthly household incomes of less than Singapore $\$ 5000$ (£1630, €2413, \$3294). Thus, generalisation of the results to populations with higher household incomes may not be appropriate. The recent NICE evidence into practice briefing on promotion of initiation and duration of breast feeding, ${ }^{4}$ however, recommended that education and support should be targeted at women with low incomes to increase rates of exclusive breast feeding.

Our study was not powered to study the differences in the breastfeeding practice among the different ethnic groups. Exploration of race or ethnicity would be useful and may help to determine whether specific subpopulations would benefit differentially from the interventions. This would allow better planning and allocation of resources used for promotion of breast feeding. We also did not examine the women's satisfaction with respect to the various interventions.

\section{Other research}

Available literature on the efficacy of interventions to improve rates of exclusive breast feeding is limited and controversial. Although professional lactation support can improve the duration of overall breast feeding, its effect in improving exclusive breast feeding is unclear. ${ }^{11822}$ Thus far, studies that report improvement of rates of exclusive breastfeeding have involved mainly community based peer counselling strategies. ${ }^{23-25}$ Even then, a randomised trial in the UK recently cast doubt on the efficacy of this approach. $^{26}$ There are current recommendations from NICE for the UK-wide implementation of the baby friendly initiative. ${ }^{4-6}$ The 2006 NICE costing report on routine postnatal care of women and their babies estimates that efforts to improve rates of breast feeding will result in substantial cost savings for the NHS. ${ }^{6}$

A randomised trial in Brazil that compared a hospital based protocol (similar to the baby friendly hospital initiative) with another incorporating intensive home visits, however, found that while the protocol achieved high rates of exclusive breast feeding in hospital, the rates fell rapidly thereafter. ${ }^{27}$ These findings were confirmed in the UK by the millennium cohort study, ${ }^{5}$ and the authors recommended that the baby friendly hospital initiative as a strategy for promotion of breast feeding should be reassessed and that other strategies are required to support mothers in the UK to breast feed for the recommended duration. ${ }^{527}$ Although combined antenatal education and postnatal support is ideal, this may be limited by economic or time resources. In one study, prenatal lactation consultant sessions lasted a mean of 111 minutes and postnatal lactation consultations with each woman lasted 139 minutes. $^{19}$

Our findings may be applied in most hospital settings to devise policies regarding strategies to promote breast feeding. Lack of breast feeding is significantly associated with higher use and cost of health care. ${ }^{28}$ Improved short and long term health of breastfed children, improved wellbeing of mothers who have breast fed, and the cost of goods consumed are major factors leading to economic benefits from the promotion of breast feeding. ${ }^{629-31}$ Future research should compare the specific cost effectiveness of such strategies for improvement of breastfeeding practice.

We thank Su-Yin Lee and the NUH-NUS Medical Publications Support Unit for help in preparing the manuscript.

Contributors: Y-SC and L-LS developed the study concept and design, wrote grant applications, supervised the study, interpreted the results, and wrote the paper with help from YSC and MR. Y-SC is guarantor. DF and K-TT advised on the study design, coordinated the study, liaised with participants, entered data, and monitored the trial. Y-HC and FSPN advised on the selection and conduct of statistical tests, and the interpretation of the results. All authors contributed to and approved the final draft.

Funding: National Healthcare Group (grant No NHG-RPR 03002).

Competing interests: None declared.

Ethical approval: Institutional Review Board of the Yong Loo Lin School of Medicine, National University of Singapore.

Provenance and peer review: Non-commissioned, externally peer reviewed.

1 World Health Organization. The optimal duration of exclusive breastfeeding: report of an expert consultation. Geneva: WHO, 2001. 


\section{WHAT IS ALREADY KNOWN ON THIS TOPIC}

Various forms of education on breast feeding are effective but only by increasing rates of initiation of breast feeding

While there is evidence for the effectiveness of professional lactation support in prolonging duration of breast feeding, the strength of its effect on the rate of exclusive breast feeding is unclear

\section{WHAT THIS STUDY ADDS}

Hospital based antenatal education on breast feeding and postnatal lactation support both significantly improve rates of exclusive breast feeding for up to six months after birth Postnatal lactation support is marginally more effective than antenatal education

www.who.int/childadolescent-health/New_Publications/ NUTRITION/WHO CAH_01_24.pdf.

2 Gartner LM, Morton J, Lawrence RA, Naylor AJ, O'Hare D, Schanler RJ, et al. American Academy of Pediatrics Section on Breastfeeding. Breastfeeding and the use of human milk. Pediatrics 2005;115:496-506.

3 Radford A, Rickitt C, Williams A. Breast feeding: the baby friendly initiative. Unicef's baby friendly initiative is making great progress in UK. BMJ 1998;317:1385.

4 Dyson L, Renfrew M, McFadden A, McCormick F, Herbert G, Thomas I. Promotion of breastfeeding initiation and duration. Evidence into practice briefing. London: NICE, July 2006. www.nice.org.uk/ download.aspx $? 0=346169$ \&template $=$ download.aspx

5 Bartington S, Griffiths LJ, Tate AR, Dezateux C, the Millennium Cohor Study Health Group. Are breastfeeding rates higher among mothers delivering in Baby Friendly accredited maternity units in the UK? Int Epidemiol 2006;35:1178-86.

6 National costing report: routine postnatal care of women and their babies. NICE Clinical Guideline No 37, July 2006. www.nice.org.uk/ download.aspx?o=345136\&template=download.aspx

7 Foo LL, Quek SJ, Ng SA, Lim MT, Deurenberg-Yap M. Breastfeeding prevalence and practices among Singaporean Chinese, Malay and Indian mothers. Health Promot Int 2005;20:229-37.

8 Mattar CN, Chong YS, Chan YS, Chew A, Tan P, Chan YH, et al. Simple antenatal preparation to improve breastfeeding practice: a randomized controlled trial. Obstet Gynecol 2007;109:73-80.

9 Memmott M, Bonuck K. Mother's reactions to a skills-based breastfeeding promotion intervention. Matern Child Nutr 2006;2:40-50.

10 Hoddinott $P$, Pill R. Qualitative study of decisions about infant feeding among women in east end of London. BMJ 1999;318:30-4.

11 Sikorski J, Renfrew MJ, Pindoria S, Wade A. Support for breastfeeding mothers. Cochrane Database Syst Rev 2002;(1):CD001141.

12 Dyson L, McCormick F, Renfrew MJ. Interventions for promoting the initiation of breastfeeding. Cochrane Database Syst Rev 2005;(2): CD001688.

13 National University Hospital, Breastfeeding Support Group. Breastfeeding: natural is best. 1st ed. Singapore: National University Hospital, 2002.
14 Danner S, Cerruti E. Nursing your baby for the first time. New York: Childbirth Graphics, 1998.

15 Lee J. Odds ratio or relative risk for cross-sectional data? Int J Epidemiol 1994;23:201-3.

16 Oddy WH, Sly PD, de Klerk NH, Landau LI, Kendall GE, Holt PG, et al. Breast feeding and respiratory morbidity in infancy: a birth cohort study. Arch Dis Child 2003;88:224-8.

17 Raisler J, Alexander C, O'Campo P. Breast-feeding and infant illness: a dose-response relationship? Am J Public Health 1999;89:25-30.

18 Heinig MJ. Host defense benefits of breastfeeding for the infant. Effect of breastfeeding duration and exclusivity. Pediatr Clin North Am 2001;48:105-23.

19 Bonuck KA, Trombley M, Freeman K, McKee D. Randomized, controlled trial of a prenatal and postnatal lactation consultant intervention on duration and intensity of breastfeeding up to 12 months. Pediatrics 2005;116:1413-26.

20 Hamlyn B, Brooker S, Oleinikova K, Wands S. Infant feeding 2000. A surveyconducted on behalf of the Department of Health, the Scottish Executive, the National Assembly of Wales and the Department of Health, Social Services and Public Safetyin Northern Ireland. London: Stationery Office, 2002.

21 Kramer MS, Chalmers B, Hodnett ED, Sevkovskaya Z, Dzikovich I, Shapiro S, et al. Promotion of breastfeeding intervention trial (PROBIT). JAMA 2001;285:413-20.

22 Bonuck KA, Freeman K, Trombley M. Randomized controlled trial of a prenatal and postnatal lactation consultant intervention on infant health care use. Arch Pediatr Adolesc Med 2006;160:953-60.

23 Morrow AL, Guerrero ML, Shults J, Culva J, Lutter C, Bravo J, et al. Efficacy of home-based peer counselling to promote exclusive breastfeeding: a randomised controlled trial. Lancet 1999;353:1226-31.

24 Bhandari N, Bahl R, Mazumdar S, Martines J, Black RE, Bhan MK, Infant Feeding Study Group. Effect of community-based promotion of exclusive breastfeeding on diarrhoeal illness and growth: a cluster randomised controlled trial. Lancet 2003;361:1418-23.

25 Haider R, Ashworth A, Kabir I, Huttly SR. Effect of community-based peer counsellors on exclusive breastfeeding practices in Dhaka, Bangladesh: a randomised controlled trial. Lancet 2000;356:1643-7.

26 Graffy J, Taylor J, Williams A, Eldridge S. Randomised controlled trial of support from volunteer counsellors for mothers considering breast feeding. BMJ 2004;328:26.

27 Coutinho SB, de Lira PIC, de Carvalho Lima M, Ashworth A. Comparison of the effect of two systems for the promotion of exclusive breastfeeding. Lancet 2005;366:1094-100.

28 Cattaneo A, Ronfani L, Burmaz T, Quintero-Romero S, Macaluso A, Di Mario S. Infant feeding and cost of health care: a cohort study. Acto Paediatr 2006;95:540-6.

29 Weimer J. The economic benefits of breast feeding: a review and analysis. Washington, DC: Economic Research Service, US Department of Agriculture, 2001-3 (Report No 13:1-20).

30 Ball TM, Wright AL. Health care cost of formula-feeding in the first year of life. Pediatrics 1999;103:870-6.

31 Fok D, Mong TG, Chua D. The economics of breastfeeding in Singapore. Breastfeed Rev 1998;6:5-9.

Accepted: 28 June 2007 